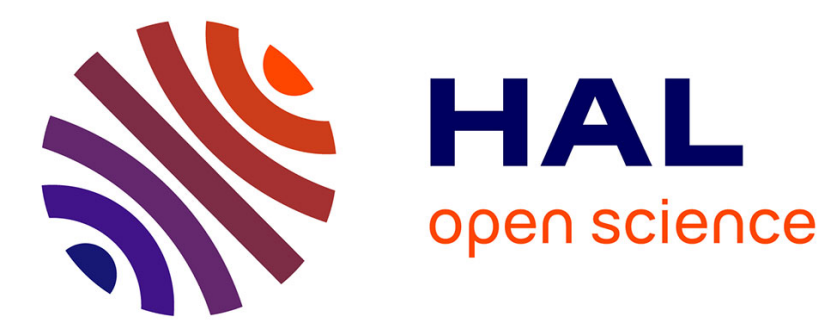

\title{
Computing floating-point logarithms with fixed-point operations
}

Julien Le Maire, Nicolas Brunie, Florent de Dinechin, Jean-Michel Muller

\section{To cite this version:}

Julien Le Maire, Nicolas Brunie, Florent de Dinechin, Jean-Michel Muller. Computing floating-point logarithms with fixed-point operations. 23rd IEEE Symposium on Computer Arithmetic, IEEE, Jul 2016, Santa Clara, United States. hal-01227877

\section{HAL Id: hal-01227877 \\ https://hal.science/hal-01227877}

Submitted on 12 Nov 2015

HAL is a multi-disciplinary open access archive for the deposit and dissemination of scientific research documents, whether they are published or not. The documents may come from teaching and research institutions in France or abroad, or from public or private research centers.
L'archive ouverte pluridisciplinaire HAL, est destinée au dépôt et à la diffusion de documents scientifiques de niveau recherche, publiés ou non, émanant des établissements d'enseignement et de recherche français ou étrangers, des laboratoires publics ou privés. 


\section{Computing floating-point logarithms with fixed-point operations}

\author{
Julien Le Maire \\ Univ Lyon, ENS Lyon
}

\author{
Nicolas Brunie \\ Kalray
}

\author{
Florent de Dinechin \\ Univ Lyon, INSA Lyon, Inria, CITI
}

Jean-Michel Muller

Univ Lyon, CNRS, Inria, LIP

\begin{abstract}
Elementary functions from the mathematical library input and output floating-point numbers. However it is possible to implement them purely using integer/fixed-point arithmetic. This option was not attractive between 1985 and 2005 , because mainstream processor hardware supported 64-bit floating-point, but only 32-bit integers. Besides, conversions between floatingpoint and integer were costly. This has changed in recent years, in particular with the generalization of native 64-bit integer support. The purpose of this article is therefore to reevaluate the relevance of computing floating-point functions in fixed-point. For this, several variants of the double-precision logarithm function are implemented and evaluated.

Formulating the problem as a fixed-point one is easy after the range has been (classically) reduced. Then, 64-bit integers provide slightly more accuracy than 53-bit mantissa, which helps speed up the evaluation. Finally, multi-word arithmetic, critical for accurate implementations, is much faster in fixed-point, and natively supported by recent compilers. Novel techniques of argument reduction and rounding test are introduced in this context.

Thanks to all this, a purely integer implementation of the correctly rounded double-precision logarithm outperforms the previous state of the art, with the worst-case execution time reduced by a factor 5 . This work also introduces variants of the logarithm that input a floating-point number and output the result in fixed-point. These are shown to be both more accurate and more efficient than the traditional floating-point functions for some applications.
\end{abstract}

Index Terms-floating-point, fixed-point, elementary function, logarithm, correct rounding

\section{INTRODUCTION}

Current processors essentially support two families of native data-types: integer, and floating-point. In each family, several variants are supported.

Integer sizes are typically 8 and 16 bits in low-power controllers such as MSP430, and 8, 16 and 32 bits for middle-range embedded processors such as the ARM family. Workstation processors and high-end embedded ones have recently added native 64-bit integer support, essentially to address memories larger than $2^{32}$ bytes $(4 \mathrm{~Gb})$.

Floating-point was standardized in 1985 with two sizes, 32 and 64 bits. A 80-bit format is supported in the x86 and IA64 families. The 2008 revision of the IEEE-754 standard [1] has added a 128-bit format, currently unsupported in hardware.

To compute on reals, floating-point numbers are the preferred format. The main reason for this is their ease of use, but performance is another compelling reason: until recently, native support for floating-point was more accurate (64-bit versus 32). Therefore, to compute on precisions larger than 32

\begin{tabular}{llc}
$\stackrel{1960}{1}$ & 1980 & 2000 \\
\hline $\begin{array}{l}\text { floating-point } \\
\text { integer }\end{array}$ & 32-bit & IEEE-754: 64-bit \\
& & 64-bit
\end{tabular}

Figure 1: Arithmetic capabilities of mainstream processors

bits, using floating-point was faster than using integers (Figure 1). Indeed, the floating-point units and memory subsytems of workstations processors are designed to achieve maximum floating-point performance on essential numerical kernels, such as the vector product around which all linear algebra can be built. At the time of writing this article, it remains true that a high-end processor can achieve more floating-point operations than integer operations per seconds [2]. This is mainly due to the wide vector units (such as Intel's AVX extensions) not fully supporting 64-bit integer multiplication.

However, integer computations are inherently simpler than floating point. For instance, integer addition takes one cycle (actually it more or less defines the processor cycle). Conversely, floating-point addition involves exponent processing, shifts and leading-zero counts: it typically requires 3 to 6 cycles.

Besides, integers may provide more accuracy in situations where the range of the data is well framed: in such cases the exponent is known, and its bits in a floating-point representation are therefore wasted.

One such situation is the the implementation of an elementary function from the standard mathematical library (libm). There, the programmer has a very good understanding of the ranges of all the intermediate data. Nevertheless, almost all the current implementations of elementary function use internal floating-point formats, probably because of the performance gap shown in Figure 1. The objective of the present work is to design an integer-only implementation of an elementary function, and discuss the effort involved and the resulting performance.

The logarithm was chosen for two reasons. The first is that it is one of the easiest functions to implement, with a large body of literature and open-source implementations to build upon, and a reasonably simple range reduction. The second was to study a floating-point-in, fixed-point-out variant that will be motivated and detailed in Section VI.

A remarkable conclusion is that the implementation effort 
is comparable or lower than for an equivalent floating-point implementation. Once the proper tools are set up (reviewed in Section II), reasoning on integers is actually easier than reasoning on floating-point. Proving code is even easier.

A second remarkable conclusion is that the performance is well on par with floating-point implementations for 64-bit computations. Besides, as soon as intermediate computations require double-word arithmetic, integers offer large speedups over floating-point. This is the case for correctly-rounded functions [3]: the present work demonstrates a worst-case performance improvements by a factor 5 over the previous state of the art. This worst-case time is even better than the average time of the default GNU/Linux log function.

The remainder of this article is organized as follows. Section II presents in more details fixed-point arithmetic, the art of representing reals using integers. Section III introduces the variants of the double-precision logarithm that are studied in this work. Section IV presents the algorithm used, with a focus on new techniques in the integer context. Section $\mathrm{V}$ presents and analyses performance results. Section VI advocates, with an application case study, a new interface to the 64-bit logarithm, inputting a floating-point number but outputting a fixed-point result. Section VII concludes.

\section{FloATING-POINT VERSUS FIXED-POINT ARITHMETIC}

\section{A. IEEE-754 floating-point}

Floating-point numbers are commonly used in computer science as an easy-to-use and accurate approximation for real numbers. Binary floating-point computing was first introduced in the Zuse Z2 electro-mechanical computer in the 1940s. A sign bit $s$, an integer exponent $e$ and a fraction $f \in \pm[1,2[$ are used to represent the number $(-1)^{s} \cdot 2^{e} \cdot f$.

In the beginning of the computer era, a lot of different floating-point formats were used in various platforms. This improved in 1985, with the adoption of the IEEE-754 Standard for Floating-Point Arithmetic. This standard defined several floating-point formats, the main ones being a 32-bit format and a 64-bit format respectively called binary 32 and binary64 in the following. More importantly, it also precisely defined the behaviour of the basic operations on these formats. The standard mandates that an operation must return floating-point number uniquely defined as the exact result, rounded according to a well-specified rounding mode. The 2008 revision of the standard, among other things, included specifications of the most used elementary functions, such as sin, tan, exp, log, $\log _{10} \ldots$ However, correct rounding is only recommended for them, due to its perceived high performance overhead. A contribution of this article is to reduce this overhead.

\section{B. Generalities on fixed-point arithmetic}

In a fixed-point program, each variable is an integer scaled by a constant exponent. This exponent is implicit: it is not stored in the number itself, contrary to the exponent of a floating-point number. Therefore, it has to be managed explicitely. For instance, to add two variables that have different implicit exponents, an explicit shift must be inserted in the program before the integer addition in order to align both fixed points (see Figure 2). Thus, implementing an algorithm in fixed point is more complex and error-prone than implementing the same algorithm in floating point:

- It requires a good understanding of the orders of magnitude of all the variables, in order to decide where their fixed point should be.

- There is no representation of infinities as in floating-point. Possible overflows have to be predicted and explicitely managed.

- Integer numbers may be signed (using two's complement), or not.

- The cheapest rounding mode available is truncation (which can be achieved by shifting right or or masking). This corresponds to a rounding toward $-\infty$, both for signed and unsigned arithmetic. Rounding to the nearest is achieved by $\circ(x)=\lfloor x+1 / 2\rfloor$ at the cost of an addition.

- When multiplying two integers of the same size, the size of the result is twice the size of the inputs. If the inputs were fractional fixed-point numbers, the high part of the product (which holds the most significant bits) is needed, as illustrated by Figure 3.

\begin{tabular}{|c|c|}
\hline $\begin{array}{r}0 \times 0.00001234 \\
+\quad 0 \times 0.00567890 \\
=0 \times 0.00568 \mathrm{AC4}\end{array}$ & $\mid \begin{array}{l}\text { uint32_t } a=0 \times 1234 ; \\
\text { // exponent: }-32 \\
\text { uint } 32 \_t \mathrm{~b}=0 \times 56781 ; \\
\text { // exponent: }-28 \\
\text { uint32_r } \mathrm{r}=\mathrm{a}+(\mathrm{b}<<4) \text {; }\end{array}$ \\
\hline (a) Math. view & (b) Source code in $\mathrm{C}$ \\
\hline
\end{tabular}

Figure 2: Addition of two fixed-point numbers with different implicit exponents.

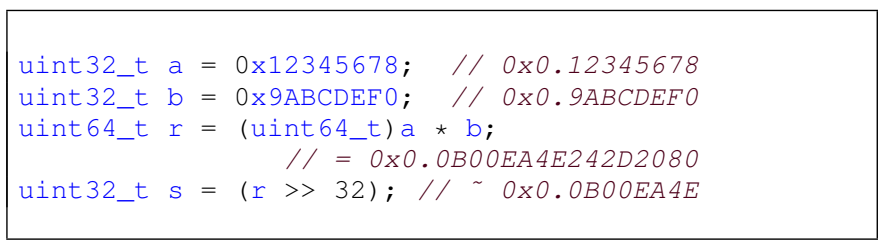

Figure 3: Multiplication of two fixed-point numbers.

\section{Support of high fixed-point precisions in current hardware and compilers}

A limited set of primitives are needed to efficiently implement floating-point elementary functions in fixed-point: addition and multiplication, boolean operations (to implement bit masks, etc), but also leading zero count and shifts (to convert between fixed-point and floating-point). Processor instructions exist to perform most of these tasks. On the x8464 architecture [4], [5], they are available on 64 bits natively, and can be chained efficiently to operate on 128-bit data. For instance, 


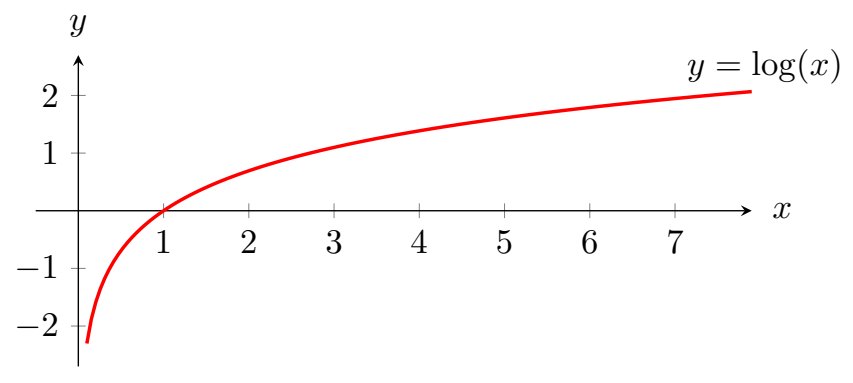

Figure 4: The natural logarithm function

- a 128-bit addition can be performed in two instructions operating on 64-bit data: an add, and an adc (add with carry);

- the full multiplication (of two 64-bit values into a 128-bit one) is done with the mulq assembler instruction;

- The lzcnt instruction performs leading zero count.

What is more, the integer datatypes _int128_t and uint128_t, while not belonging to standard $C$, are supported by most mainstream compilers (GCC, ICC, Clang). The addition in $\mathrm{C}$ of two _ int 128_t will generate the sequence of add and adc previously mentioned. A full multiplication of two 64-bit integers can be achieved by casting operands to the types _uint128_t and _uint128_t, similarly to Figure 3. The generated code will only consist of one mulq instruction. We define the macro fullmul for this (it inputs two int64 and returns an int128), and we also define a macro highmul (that returns the high 64 bits only).

Finally, instructions that are not exposed in the $\mathrm{C}$ syntax (such as add-with-carry or leading-zero-count) can be accessed thanks to intrinsics which are also de-facto standard. For example, in GCC, ICC and CLang, 128-bit leading zero count can be achieved with the builtin function _builtin_clzll.

To sum up, writing efficient code manipulating 64-bit and 128-bit fixed-precision numbers is possible in a de-facto standard way.

\section{VARIANTS OF THE LOG FUNCTION}

The logarithm function, along with the exponential, it is one of the most useful elementary functions. It is continuous and monotonically increasing, and also has the following mathematical property:

$$
\log (x \cdot y)=\log (x)+\log (y) \quad .
$$

Its output range is much smaller than the input range: for any binary64 $\left.x,|\log (x)|<2^{10}\right)$. Therefore, it is easy to define fixed-point formats that capture the whole output range of a logarithm function. Such formats have 11 bits to the left of the fixed-point (including a sign bit).

Therefore, the core of our algorithm will first compute an approximation of $\log (x)$ on such a format (with the fraction size depending on the variant studied). It will then round this approximation to the nearest floating-point number.
However, an option is also to skip this final rounding. Section VI shows an application case study where this improves both performance and accuracy.

\section{A. Correctly rounded logarithm}

Modern implementation of elementary functions are at least faithful, i.e. they return one of the two floating-point numbers surrounding the exact value $\log (x)$ [6], [7], [8]. To achieve this, it is enough to compute an approximation with a confidence interval smaller than the difference between two consecutive floating-point numbers (Figure 5). This requires to use an internal format of slightly higher precision than the destination floating-point format.

Correct rounding is more difficult to achieve. In the cases when the confidence interval includes a rounding boundary, the correctly rounded value can not be decided (Figure 5, bottom). To ensure correct rounding, one has to make sure that the exact result $y=f(x)$, which can be anywhere in the confidence interval, is on the same side of the midpoint as the computed approximation.

Ziv's algorithm [9] (whose reference implementation forms the core of the libm in the GNU/Linux standard library glibc) recomputes in such cases the function with increasing accuracy, reducing the confidence interval until it is completely one one side of the rounding boundary. As such recomputations are rarely needed, this algorithm is fast in average. However it may take a lot of time in the worst case, when the actual solution is very close to a mid-point between two consecutive floating-point numbers (Figure 5).

Lefèvre and Muller computed the worst-case precision needed for certain functions (including the logarithm) [10]. This allowed the CRLibm implementation to evaluate the function in two steps only: one quick phase, and one slower phase that is always accurate enough for correct rounding.

\section{B. Classical two-phase correctly-rounded logarithm}

The present work uses the CRLibm two-phase approach:

- In the first (quick) phase, an approximation is computed (in a 128-bit integer) with an absolute accuracy of $2^{-63}$. This is enough to return correct rounding in most cases. Otherwise, the second phase is lauched.

- The second (accurate) phase computes to an absolute accuracy of $2^{-118}$ which, according to [10, p. 452] is enough to ensure correct rounding for all floating-point inputs.

\section{Variants of the binary64 logarithm function}

From this two-phase implementations, several variants can be derived.

- A single-phase correctly-rounded, IEEE-754-2008 compliant variant, essentially consisting of the accurate phase only.

- A single-phase, accurate-faithful variant, essentially consisting of the quick phase only. This version is currently not implemented properly in this work, for reasons discussed in the sequel. 


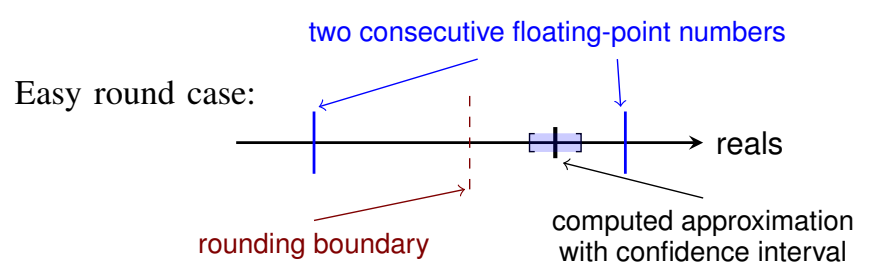

Hard round case:

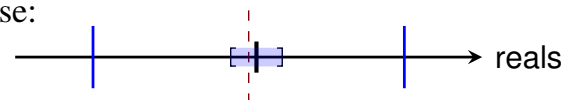

Figure 5: Rounding to the nearest an accurate approximation.

- A floating-point in, fixed-point out $\log$, outputting an int 64_t with an absolute accuracy of $2^{-52}$, essentially consisting of the first phase without the final conversion to floating-point.

- A floating-point in, fixed-point out $\log$ outputing an int128_t with an absolute accuracy of $2^{-116}$, essentially consisting of the second phase only without final conversion to floating-point.

The last to functions will be justified by an application case study in Section VI.

All these variants are actually simplifications of the twophase correctly rounded implementation, whose algorithm is presented now.

\section{Algorithm AND IMPLEMENTATION}

The algorithm can be decomposed into the following steps:

\section{A. Filtering the special cases}

The following special cases must be handled following the IEE-754 standard [1].

- input is negative or zero

- input is $\pm \infty$

- input is $\mathrm{NaN}$

- input is a subnormal number

One test (predicated false) is enough to detect all thoses cases [11]. It correspond to the case where the bit sign is set, or the exponent is either the min or max value it can take.

As this test is performed on the binary representation of the input, the algorithm begins with a copy of the binary representation of $x$ into an int 64_t variable.

On all these special cases, the function returns a special value, except for subnormals: these are normalized and the input exponent is initialized accordingly. Then the control flow gets back to the main path, where a normal input is decomposed into its fraction $x=1+2^{-52} f_{52} \in[1 ; 2)$ and exponent $E$.

\section{B. Argument range reductions}

The first range reduction simply decomposes the elements of the input:

$$
\begin{aligned}
\text { input } & =2^{E} \times x \\
\log (\text { input }) & =E \times \log (2)+\log (x)
\end{aligned}
$$

When computing $E \times \log (2)+\log (x)$, there is a possible catastrophic cancellation when $x$ is close to 2 and $E=-1$, i.e. when the input is very close to (but smaller than) 1 . To avoid it, most floating-point implementations [6], [3] first re-center the fraction around 1, using a variant of the following test: if $x>1.4$ then $x=x / 2$ and $E=E+1$. We currently choose to skip this classical recentering. This saves the cost of this test itself, but also a few additional operations in the subsequent range reductions, where round to nearest would be needed instead of truncation. The trade-off is that the accurate phase will be launched in case of a massive cancellation. This design choice, initially motivated by simplicity, is a posteriori justified by the low overhead of the accurate phase in the present implementation. However its relevance will be discussed in the conclusion.

The constant $\log (2)$ can be stored to arbitrary accuracy as several 64-bit chunks. This, as well as the computation of $E \times \log (2)$, will be detailed in Section IV-D.

Let us now address the computation of $\log (x)$ with $x \in$ $[1,2)$. This interval can be further reduced by an algorithm due to Tang [12]. The main idea is to read, from a table addressed by the $k$ most significant bits of $x$, two values: inv $v_{x} \approx \frac{1}{x}$ and $\log \left(i n v_{x}\right)$. Then if we compute $y=i n v_{x} \cdot x$, the logarithm can be computed as $\log (x)=\log (y)-\log \left(i n v_{x}\right)$.

The closer $i n v_{x}$ is to $\frac{1}{x}$, the closer $y$ will be to 1 . Indeed, if $\left|i n v_{x}-\frac{1}{x}\right| \leq \epsilon$ then:

$$
\begin{aligned}
|y-1| & \leq \mid \text { inv }_{x} \cdot x-1 \mid \\
& \leq \mid \text { inv }_{x}-\frac{1}{x} \mid \cdot x \\
& \leq \epsilon \cdot x \\
& \leq 2 \cdot \epsilon
\end{aligned}
$$

Since the range of $y$ is much smaller than the range of $x$, computing $\log (y)$ will be easier than computing $\log (x)$.

In detail, let $x_{1}$ the value of $x$ taking only in consideration its $k$ leading bits. The value stored is inv $v_{x}=\left\lceil 1 / x_{1}\right\rceil$, in order to ensure $y \geq 1$. This choice ensures that we can use only unsigned arithmetic in the sequel. However, an alternative using signed arithmetic should be tried in the near future.

It is desirable to compute $y=i n v_{x} \cdot x$ exactly. In our case this is easy for two reasons. Firstly $x$ is a binary64 mantissa (53 bits) and we work with 64-bit integers. Therefore, with a value of $i n v_{x}$ on 11 bits, the product can be computed exactly

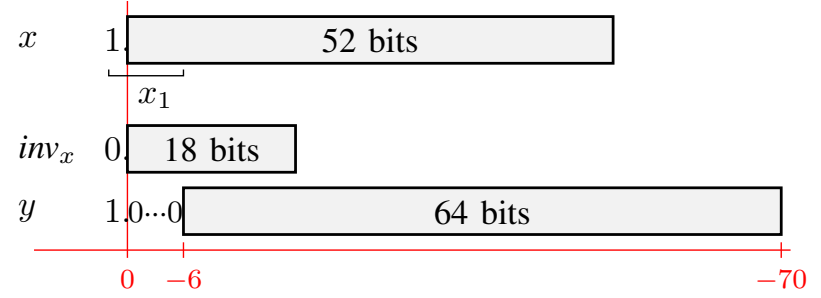

Figure 6: Tang's argument reduction, here for $k=7$.

The gray boxes are the values represented in the program. 


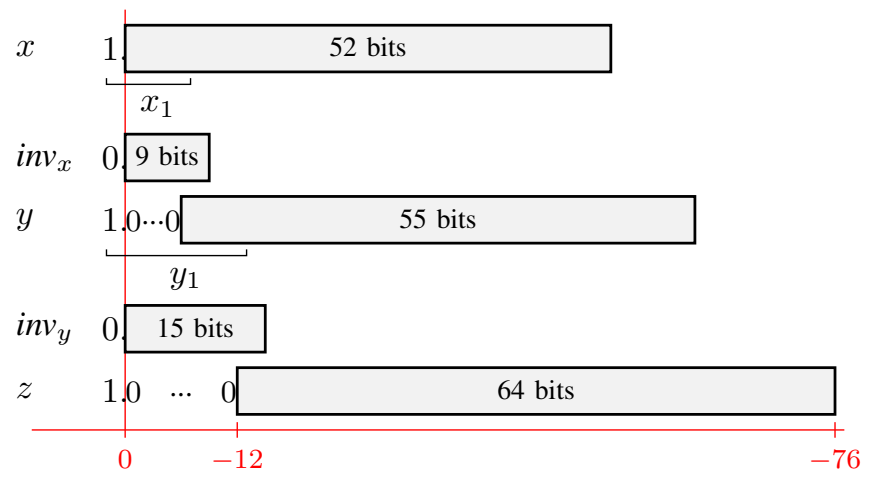

Figure 7: Two iterations of Tang's argument reduction.

\begin{tabular}{|r|c|c|}
\hline Table size (bytes) & degree 1st & degree 2nd \\
\hline 39,936 & 3 & 5 \\
12,288 & 3 & 6 \\
$\mathbf{4 , 0 3 2}$ & $\mathbf{4}$ & $\mathbf{7}$ \\
2,240 & 4 & 8 \\
2,016 & 4 & 9 \\
900 & 5 & 10 \\
594 & 6 & 12 \\
298 & 7 & 14 \\
\hline
\end{tabular}

Table I: A few Pareto points of the design space

in the low part of a 64-bit product. Secondly, the $k$ leading bits of $y$ are known, as illustrated by Figure 6, so their overflow in the high part of the product is harmless: for $k=7$, it is actually possible to approximate $i n v_{x}$ on 18 bits and still compute $y-1$ exactly in a 64-bit integer.

Thanks to this, it is possible to do this argument range reduction twice. First, the 7 leading bits of $x$ (excluding the constant 1 ) are used as an index in a table that provides inv and $\log \left(i n v_{x}\right)$. Then, $y-1$ has its 6 most significants bits being zeros (more precisely $y \in\left[0,2^{-6.41504}\right]$ ). Its 7 next bits are used as an index in a table that provides $i n v_{y}$ as well as $\log \left(i n v_{z}\right)$. Now $z=i n v_{y} \times y$ is even closer to 1 than $y$ : $z \in\left[1,1+2^{-12.6747}\right]$.

This argument reduction is depicted on Figure 7. With a total of $2^{8}$ table entries only, it achieves a similar reduction as the single-step approach would with a $2^{13}$-entry table. The delay of the second table read is more than compensated by the reduction in polynomial degree (from 7 to 4 in the quick phase and from 13 to 7 in the accurate phase).

This is an old idea in a hardware context [13], [14], [15]. It cannot be used efficiently to implement a binary64 log using binary64 arithmetic, because the latter cannot hold $z$ (nor $z-1$ ) exactly. A contribution of this work is to show that this idea is relevant when 64-bit integer arithmetic is used.

Table I shows that other trade-off between table size and polynomial degree would be possible.

The proof of these three steps of argument reduction was developed using Gappa [16]. We note $t=z-1$, and we now address the computation of $\log (1+t)$.

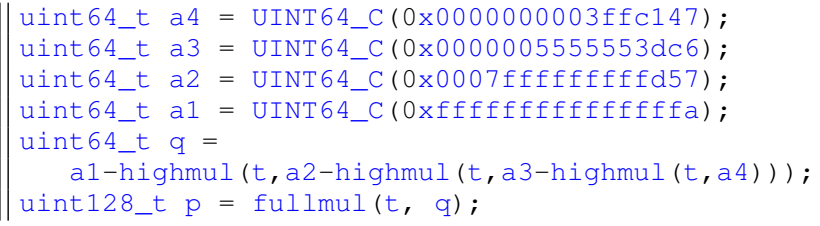

Figure 8: Shift-less polynomial evaluation

\section{Polynomial approximation and evaluation}

In each phase, the function $\log (1+t)$ may be approximated by a polynomial $P(t)$ of small degree (4 in the quick phase, 7 in the accurate phase). Thanks to the Taylor formula $\log (1+$ $t) \approx t-t^{2} / 2 \cdots$, its constant coefficient can be forced to $0: P(t)=t Q(t)$. This polynomials is computed using the fpminimax command of the Sollya tool [17]. At runtime, it is evaluated using Horner's method.

This Horner evaluation can be implemented with no runtime shift, as shown on Figure 8. Instead, the approximation polynomial was constrained to include all the shifts within the coefficients themselves. More precisely, Sollya's fpminimax can be constrained to compute the optimal polynomial among those which will avoid any shift. This lead to coefficients with leading zeroes, which may seem a waste of coefficient bits. However, we observed that this provided almost the same accuracy as a polynomial where each coefficient is stored on exactly 64 bits, in which case shifts are needed during the evaluation. In other words, removing the shifts is for free in terms of approximation accuracy.

In the quick phase, the evaluation of $Q(t)$ is performed completely in 64-bit arithmetic (using the highmul primitive). Only the final multiplication by $t$ needs a full multiplication. As $t$ itself is exact, this last multiplication is exact, and the 128-bit result will therefore always hold almost 64 significant bits after the leading non-zero bit.

In the accurate phase, all the coefficients $a_{i}$ of $Q$ (except $a_{7}$ ) must be stored in 128-bit integers. However the evaluation remains quite cheap, because $t$ remains a 64-bit integer. Specifically, Horner step $i$ computes $q_{i}=a_{i}+t \times q_{i-1}$ where the $q_{i}$ and $a_{i}$ are 128-bit. This rectangular multiplication is implemented as

$q=a i-($ fullmul $(t, H I(q))+\operatorname{highmul}(t, L O(q)))$; Again, there is no shift needed here.

\section{Result reconstruction}

Finally, the $\log$ will be evaluated as

$$
\log (\text { input })=E \log (2)-\log \left(i n v_{x}\right)-\log \left(i n v_{y}\right)+P(t) .
$$

There, $-\log \left(i n v_{x}\right)$ and $-\log \left(i n v_{y}\right)$ are precomputed values. Thus, we can choose to decompose and align them the way we want. The best is probably to align them to the format we want for the results, so they can be added without shift (Figure 9).

The value of $E \log (2)$ is computed by multiplying $E$ with slices of the binary writing of $\log (2)$. These slices are 
chosen in such a way that the product will be aligned to the format of the result: $\log (2)$ is decomposed into $\log (2)_{-1,-53}$, $\log (2)_{-54,-118}, \log (2)_{-119,+\infty}$.

The product of $\log (2)_{-1,-53}$ with $E$ (a 11-bit number) fits on a 64-bit integer: it requires only the low part of a 64-bit multiplication, and will be aligned to the high word of the result format (see figure 9).

The product of $\log (2)_{-54,-118}$ requires a fulmul (it needs more than 64 bits), but the obtained 128-bit result is also aligned. These two first terms are used in the quick phase.

Only 11 more bits of $E \log (2)$ are needed for the accurate phase, but the corresponding term needs to be shifted to place. This will be done only in the accurate phase.

The approximation $P(t)$ of $\log (z)$ is finally added. Its implicit exponent is fixed by the fact that we want $t$ exact and fitting on one int64: we therefore have to shift $t Q(t)$ before adding it.

One nice thing about fixed-point arithmetic is that this whole summation process is exact, therefore associative. The order in which it is written is not important, and this is a big difference compared to floating-point, where one has to think carefully about the parenthesing of such sums. In principle the compiler also has more freedom to schedule it than in floating-point, but it is not clear that this is exploited.

\section{E. Rounding test}

Floating-point-based correctly-rounded functions use a multiplication-based test due to Ziv, but documented much later [18]. Considering the previous summation, it is possible here to use a much simpler test. Upper bounds of the errors of each summand are added together to form an upperbound $\epsilon$ of the distance between our final sum $S$ and the actual value of the logarithm. Then, at runtime, it is enough to check if $S-\epsilon$ and $S+\epsilon$ are rounded to two different floating-point numbers or not, as seen in the figure 5 .

Figure 9 show in grey the error terms we have to sum to get $\epsilon$. Most of this error summation is performed statically, only the error on $P(t)$ uses a dynamic shift, to benefit from the fact that $P(t)$ was evaluated with good relative error.

Since only sharp error bounds are added together, the value $\epsilon$ is also sharp, and so is the test.

\section{F. Accurate phase}

For the last step, the previous sum (before the addition of the polynomial) can be fully reused. The $\log \left(i n v_{x}\right)$ and $\log \left(i n v_{y}\right)$ terms were already accurate to $2^{-118}$ The accurate phase therefore only consists of adding the third part of $E \log (2)$, and recomputing a more accurate $t P(t)$ of degree 7 as already explained in Section IV-C.

\section{RESULTS AND ANALYSIS}

\section{A. Experimental protocol}

The proposed correctly rounded implementation has been proven in Gappa, tested against the hard to round cases distributed with CRLibm, and against large numbers of random values. It passes all these tests.

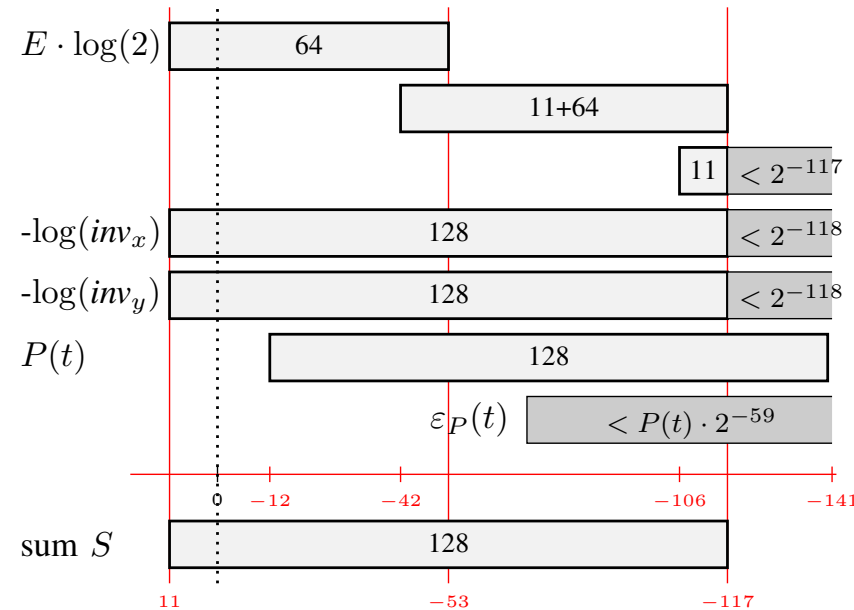

Figure 9: Solution reconstruction (error terms in darker gray)

The performance of this function is reported on two architectures: an Intel(R) Core(TM) i7-4600U CPU, and a Kalray Bostan core. The latter is a 64-bit, 4-way VLIW core with an FMA-based double-precision floating-point unit, several arithmetic and logic units, and a single 32-bits multiplier (no hardware 64-bits multiplier). A recent GCC is used in both cases (version 4.9). Experiments with ICC and CLang show no significant difference.

To mesure the performance of our function, we use standard procedures based on hardware counters ( $r d t s c$ on the Core i7, properly serialized). The functions are compiled with gcc -03 optimization. The test program is compiled with -00 to avoid unwanted optimizations. It consists of three similar loops that apply the function on an array of random data. the first calls an (externally linked) empty function, to calibrate the loop and function call overhead: all the timings are reported without this overhead. The second loop times the average case execution time of each function. The third calls the function on an array of hard-to-round cases, thus timing the worst-case execution time of the function.

The same test was run with three others implementations:

- glibc (resp newlib): the function of the GNU C standard library distributed with the Linux system on the Core i7 and Bostan respectively. The glibc uses Ziv' algorithm, and is correctly rounded.

- cr-td: the function is the CRLibm variant that only uses binary64.

- cr-de: a variant of CRLibm that uses the double extended (80 bits, with a fraction on 64 bits) format available on the $\mathrm{x} 86$ platform. Comparing to it hints to what depends on having 64 bits instead of 53, and what depends on having faster arithmetic.

Table II presents some parameters of these implementation. It is worth to mention that the proposed implementation has the smallest memory consumption. The glibc is not a twophase algorithm. It also uses a degree 3 polynomial for the case $|x-1|<0.03$ (see file u_log. $\mathrm{c}$ in the glibc sources). 


\begin{tabular}{|l|c|c|c|c|}
\hline & glibc & crlibm-td & crlibm-de & cr-FixP \\
\hline degree pol. 1 & $3 / 8$ & 6 & 7 & 4 \\
degree pol. 2 & 20 & 12 & 14 & 7 \\
tables size & $13 \mathrm{~Kb}$ & 8192 bytes & 6144 bytes & 4032 bytes \\
$\%$ accurate phase & N/A & 1.5 & 0.4 & 4.4 \\
\hline
\end{tabular}

Table II: Implementation parameters of the correctly rounded implementations.

\section{B. Timings of correctly rounded implementations}

Table III compares the speed of the available correctly rounded implementations on the Core i7 processor. For an absolute reference, it also shows what is probably the best available faithful scalar implementation of the logarithm for this processor: it is distributed in the Math Kernel Library (MKL) with Intel's own compiler icc. To obtain this measure, the same test was compiled with icc $-\mathrm{OO}$ -fp-model precise - $\mathrm{lm}$. This processor-specific implementation, written by Intel experts, probably uses tricks such as exploiting the hardware reciprocal approximation instead of a table of inverse [7], or the FMA available in recent AVX extensions [5].

The main results are the following.

- The average time is on par with the best state of the art: crlibm-de, that uses 80-bit floating-point and larger tables. It is within a factor two of the absolute reference, MKL, which is not correctly rounded.

- The worst-case time has been improved by a factor 5, and is now within a factor two of the average time.

Table IV compares these timings (broken down in their different steps) on two processors. What is interesting here is that the Bostan performance is respectable, although it only has 32-bit multiplication support.

In Table II, the proposed cr-FixP implementation is the worst on one metric: the percentage of calls to the accurate phase. This is essentially due to the cancellation problem for values slightly smaller than $1-$ the random generator used is actually biased towards this case. It would be a bad design choice to always lauch the accurate phase for such central values if the accurate phase was very slow. However, with an accurate phase within a factor two of the quick one, it

\begin{tabular}{|c||c||c|c|c|c|}
\hline cycles & $M K L$ & glibc & crlibm & cr-de & cr-FixP \\
\hline avg time & 25 & 90 & 69 & 46 & 49 \\
max time & 25 & 11,554 & 642 & 410 & 79 \\
\hline
\end{tabular}

Table III: Average and max runing time (in processor cycles) of correctly rounded implementations on Intel Core i7. Italic denotes lack of correct rounding.

\begin{tabular}{|l|c|c|}
\hline cycles & Core i5 & Bostan \\
\hline System & glibc & newlib \\
& 90 & 105 \\
\hline quick phase alone & 42 & 94 \\
accurate phase alone & 74 & 181 \\
both phases (avg time) & 49 & 121 \\
both phases (max time) & 79 & 225 \\
\hline
\end{tabular}

Table IV: Running times of each step becomes a sensible design choice. We initially felt that this choice allowed us to save a few cycles in the quick phase. This feeling can only be confirmed by the design and test of an implementation including the classical recentering and more signed arithmetic. What is already clear is that the polynomial evaluation schemes we already use will provide the required relative accuracy in this case. Therefore, when this implementation is available, running its first step only will provide a faithful result, which is not the case of the current implementation.

\section{FlOATING-POINT IN, FIXED-POINT OUT VARIANT}

Finally, Table V shows the performance of the variant that returns a 64-bit signed integer approximating $2^{52} \log (x)$. This variant performs the final summation completely in int64. Therefore it requires even less tables. Moreover, a polynomial of degree 3 is enough to achieve absolute accuracy of $2^{-59}$ which is largely enough in this context. With all this, and with the removal of the final conversion to a binary64 floatingpoint number, this function offers performance on par with the MKL.

However, the main impact of offering this variant may be in applications that compute sums of logs. An archetype of this class of applications is a DNA sequence alignment algorithm known as TKF91 [19]. It is a dynamic programming algorithm that determines an alignment as a path within a 2D array. The borders of an array are initialized with log-likelihoods, then the array is filled using recurrence formulae that involve only max and + operations.

All current implementations of this algorithm use a floatingpoint array. With the proposed $\log F i x 64$, an int64 array can be used instead. This should lead to an implementation of TKF91 that is both faster and more accurate:

- faster, not only because $\log$ Fix 64 is faster, but also because the core computation can now use int64 addition and max: both are 1-cycle, vectorizable operations;

- more accurate, because larger initialization logs impose an absolute error that can be up to $2^{-42}$, whereas $\log$ Fix64 will have a constant absolute error of $2^{-52}$.

- more accurate, also because the computation of all the additions inside the array become exact operations: they still accumulate the errors due to the initialization logs, but they do not add rounding errors themselves, as floating-point additions do.

\begin{tabular}{|c||c|c|c|c|}
\hline $\begin{array}{c}\text { output } \\
\text { format }\end{array}$ & $\begin{array}{c}\text { absolute } \\
\text { accuracy }\end{array}$ & $\begin{array}{c}\text { table } \\
\text { size }\end{array}$ & $\begin{array}{c}\text { Core i5 } \\
\text { cycles }\end{array}$ & $\begin{array}{c}\text { Bostan } \\
\text { cycles }\end{array}$ \\
\hline \hline Fix64 & $2^{-52}$ & 2304 & 24 & 66 \\
Fix128 & $2^{-116}$ & 4032 & 60 & 179 \\
\hline \hline double (libm) & $2^{-42}$ & & 90 & 105 \\
\hline
\end{tabular}

Table V: Performance of floating-point in, fixed-point out functions. Fix64 is the code of the first step only, without the conversion to float. Fix128 is the code of the second step only, without the conversion to float. 
A quantitative evaluation of these benefits on TKF91 is in progress. Of course, applications that compute exponentials of sums could similarly benefit from a fixed-point in, floatingpoint out exponential. The same also holds for the base- 2 and base-10 variants of these functions.

\section{CONCLUSION AND FUTURE WORK}

This work studies the implementation of the correctly rounded logarithm using integer operations. The main idea is to use, instead of binary64 (double-precision), the fast 64-bit integer arithmetic that is nowadays mainstream.

The main result is to improve the worst-case time of a correctly-rounded implementation by a factor 5 with respects to the state of the art, using smaller tables. This new worst case time is better than the average time of the default $\mathrm{glibc}$ $\log$ of Linux systems. It is also within a factor 4 of the best achievable faithful implementation (the MKL).

We believe that this promising result can be generalized in the following claim: as soon as double-word arithmetic is heavily needed in the implementation of an elementary function (and this is always the case for correctly rounded versions), integer arithmetic becomes very relevant, essentially because 128-bit addition is a 2-cycle operation.

Another claim is that working in fixed point is not a problem in this context: efficient elementary function algorithms always reduce their arguments to a small domain. This second claim, however, requires other experiments, with other functions, to support it. For instance, the Payne and Hanek argument reduction for trigonometric functions is a fixed-point algorithm that provides a fixed-point reduced argument.

The main limitation of this work is probably that it can currently not be vectorized: vector units do not currently offer the necessary multiplication operations. It is not unreasonable to expect that they could be added in the future. If this happens, running the accurate phase alone will provide a branchless implementation that we can expect to be within a factor 3 of the faithful vector version. The inherent test in current twophase correct-rounding approaches is a roadblock for their vectorization, and this solution would remove it completely.

Implementing a function using floating point requires acute floating-point expertise, in particular a mastering of the range and accuracy of all intermediate variables. Considering this, we are also inclined to claim that an integer implementation is in many ways simpler, for instance when one has to add many terms. Still, this is another claim that requires more experiments, because the logarithm was chosen as probably the simplest from this point of view.

A last claim of this article is that floating-point-in, fixedpoint out variants of logarithms would make sense in some applications, both from a performance and from an accuracy point of view. This work provides such a variant. It will be interesting to study if this idea may be generalized to other functions.

Several new techniques have been developed in this article. The more generally useful are the rounding test, and the shift-less polynomial evaluation. A new two-step argument reduction is specific to the logarithm.

Some short-term questions remain, the main one being the possibility to achieve high relative accuracy around 1 without degrading the performance of the quick phase. Work has begun to integrate this code the Metalibm framework [20], which will make such experiments easier.

Another observation is that the published table-maker's dilemma worst-case data is not in an ideal format for a fixedpoint implementation: it is currently only given in relative terms on a large range of exponents.

The best-of-class faithful implementation on Intel processors, MKL, still uses floating point. We have shown that the same performance can be achieved using integers only, but then there is an integer/floating-point conversions overhead that cannot be avoided. In this case, maybe the optimal twophase correctly rounded implementations should use floatingpoint in the quick phase, and integers in the accurate phase. This will add new challenges, such as sharing the tables between the phases.

\section{ACKNOWLEDGEMENTS}

This work was supported by the French National Research Agency through the INS program MetaLibm.

The authors are thankful to Alexandros Stamatakis for pointing to the TKF91 algorithm, to Sarah Lutteropp and Pierre Barbera for testing logFix64, and to C. Lauter for assistance with the test procedure.

\section{REFERENCES}

[1] "IEEE standard for floating-point arithmetic," IEEE 754-2008, also ISO/IEC/IEEE 60559:2011, Aug. 2008.

[2] C. Pernet, "Exact linear algebra algorithmic: Theory and practice," in Proceedings of the 2015 ACM on International Symposium on Symbolic and Algebraic Computation, ser. ISSAC '15. New York, NY, USA: ACM, 2015, pp. 17-18. [Online]. Available: http://lig-membres.imag.fr/pernet/Publications/issac_tuto_pernet.pdf

[3] F. de Dinechin, C. Q. Lauter, and J.-M. Muller, "Fast and correctly rounded logarithms in double-precision," Theoretical Informatics and Applications, vol. 41, pp. 85-102, 2007.

[4] Intel Corporation, Intel ${ }^{\circledR} 64$ and IA-32 Architectures Software Developer's Manual, Sep. 2015, no. 253669-056US.

[5] - Intel ${ }^{\circledR} 64$ and IA-32 Architectures Optimization Reference Manual, Sep. 2015, no. 248966-31.

[6] P. Markstein, IA-64 and Elementary Functions: Speed and Precision, ser. Hewlett-Packard Professional Books. Prentice Hall, 2000.

[7] M. Cornea, J. Harrison, and P. T. P. Tang, Scientific Computing on Itanium $^{\circledR}$-based Systems. Intel Press, 2002.

[8] J.-M. Muller, Elementary Functions, Algorithms and Implementation, 2nd ed. Birkhäuser, 2006.

[9] A. Ziv, "Fast evaluation of elementary mathematical functions with correctly rounded last bit," ACM Transactions on Mathematical Software, vol. 17, no. 3, pp. 410-423, 1991.

[10] J.-M. Muller, N. Brisebarre, F. de Dinechin, C.-P. Jeannerod, V. Lefèvre, G. Melquiond, N. Revol, D. Stehlé, and S. Torres, Handbook of FloatingPoint Arithmetic. Birkhauser Boston, 2009.

[11] C. S. Anderson, S. Story, and N. Astafiev, "Accurate math functions on the Intel IA-32 architecture: A performance-driven design," in 7th Conference on Real Numbers and Computers, 2006, pp. 93-105.

[12] P. T. P. Tang, "Table-driven implementation of the logarithm function in IEEE floating-point arithmetic," ACM Transactions on Mathematical Software, vol. 16, no. 4, pp. 378 - 400, 1990.

[13] M. Ercegovac, "Radix-16 evaluation of certain elementary functions," IEEE Transactions on Computers, vol. C-22, no. 6, pp. 561-566, 1973.

[14] W. F. Wong and E. Goto, "Fast hardware-based algorithms for elementary function computations using rectangular multipliers," IEEE Transactions on Computers, vol. 43, no. 3, pp. 278-294, 1994. 
[15] J. Detrey, F. de Dinechin, and X. Pujol, "Return of the hardware floatingpoint elementary function," in 18th Symposium on Computer Arithmetic. IEEE, 2007, pp. 161-168.

[16] F. de Dinechin, C. Lauter, and G. Melquiond, "Certifying the floating-point implementation of an elementary function using Gappa," IEEE Transactions on Computers, vol. 60, no. 2, pp. 242-253, Feb. 2011. [Online]. Available: http://ieeexplore.iee.org/xpl/articleDetails. jsp?arnumber $=5483294$

[17] S. Chevillard, M. Joldeş, and C. Lauter, "Sollya: An environment for the development of numerical codes," in Mathematical Software - ICMS 2010, ser. Lecture Notes in Computer Science, vol. 6327. Springer, Sep. 2010.

[18] F. de Dinechin, C. Lauter, J.-M. Muller, and S. Torres, "On Ziv's rounding test," ACM Transactions on Mathematical Software, vol. 39, no. 4, 2013. [Online]. Available: http://dl.acm.org/citation.cfm?id= 2491495

[19] J. Thorne, H. Kishino, and J. Felsenstein, "An evolutionary model for maximum likelihood alignment of DNA sequences," Journal of Molecular Evolution, vol. 33, no. 2, pp. 114-124, 1991. [Online]. Available: http://dx.doi.org/10.1007/BF02193625

[20] N. Brunie, F. de Dinechin, O. Kupriianova, and C. Lauter, "Code generators for mathematical functions," in 22nd Symposium of Computer Arithmetic. IEEE, Jun. 2015, best paper award. 